\title{
Effects of Interferential Current Treatment on Pain, Disability, and Balance in Patients with Chronic Low Back Pain: A Randomized Controlled Study
}

\author{
Kyoung-Sim Jung, PT $\cdot$ Tae-Sung In, $\mathrm{PT}^{\dagger}$
}

Department of Physical Therapy, Gimcheon University

Received: June 14, 2020 / Revised: June 19, 2020 / Accepted: July 12, 2020

(c) 2020 J Korean Soc Phys Med

\section{| Abstract |}

PURPOSE: This study investigated the efficacy of interferential current (IFC) treatment on the improvement of pain, disability, and balance in patients with chronic nonspecific low back pain.

METHODS: A double-blind randomized clinical trial was conducted with 40 patients with chronic nonspecific low back pain. The patients were randomly allocated into two groups: the IFC treatment group $(\mathrm{n}=20)$ and the placebo treatment group $(n=20)$. The IFC group received 30 minutes of IFC treatment on the lumbar region, while the placebo group received IFC treatment without real electrical stimulation. The intervention was administered five days a week for two weeks.

RESULTS: The primary outcomes of resting pain and pain during functional movement were measured by a visual analogue scale. The secondary measurements included the Oswestry disability index (ODI) for low back pain and

$\uparrow$ Corresponding Author : Tae-Sung In in8386@naver.com, https://orcid.org/0000-0002-7672-5150

This is an Open Access article distributed under the terms of the Creative Commons Attribution Non-Commercial License (http://creativecommons.org/licenses/by-nc/3.0) which permits unrestricted non-commercial use, distribution, and reproduction in any medium, provided the original work is properly cited. postural sway. The measurements were performed before and after the two-week intervention period. Compared to the placebo treatment group, the IFC treatment group showed significantly greater improvement in pain during anterior trunk flexion in the standing position $(\mathrm{p}=.029)$, ODI $(\mathrm{p}=$ .039), and postural sway when subjects stood with their eyes closed $(p=.010)$ at the end of the intervention.

CONCLUSION: Our findings show that IFC treatment can improve pain, disability, and postural sway, thus, highlighting the benefits of somatosensory stimulation from IFC.

Key Words: Interferential current (IFC), Pain, Disability, postural sway

\section{Introduction}

Nonspecific chronic low back pain (CLBP) is one of the four most common diseases in the world [1]. Low back pain causes a decrease in balance and walking ability as well as a degradation in the quality of life [2]. About 9 percent of balance disorders are reported to be caused by low back pain [3]. When the Center of Mass (COM) is out of balance, the body should be able to rapidly switch the Center of Pressure (COP) in the opposite direction to maintain body balance [4]. However, in patients with chronic low back pain, the location of the COM cannot 
be accurately determined due to the damage of proprioceptive sensibility, and the safety margin of adaptive COP movement for COM oscillation increases [5,6]. Treatment of low back pain by electrotherapy is a noninvasive, nonpharmacologic therapy for which transcutaneous electrical nerve stimulation (TENS) and interferential current (IFC) are predominantly used [7]. IFC treatment method uses new low-frequency $(0-250 \mathrm{~Hz})$ that crosses different intermediate frequency currents of around 4000 $\mathrm{Hz}[8]$, allowing currents to penetrate deeper tissues by reducing skin resistance $[8,9]$, which is a useful advantage over TENS [8]. By stimulating sensory nerve fibers, IFC treatment not only reduces pain but also promotes muscle relaxation and blood circulation [10,11], and stimulates proprioceptive sensibility, thus improving balance [12]. Correa et al [13]. argued that IFC treatment was effective in reducing pain in patients with chronic low back pain. In addition, when IFC treatment was applied to patients with nonspecific chronic low back pain for 2 weeks, it was reported to show improvement in disability as well as alleviating pain [14]. IFC treatment has been shown to reduce pain in several musculoskeletal disorders such as knees [15-17] and shoulders [18-20]. However, studies reporting the use of IFC treatment in patients with low back pain are scarce $[17,21,22]$, and most of the previous studies on back pain have focused on pain and dysfunction, and studies on improving balance have been very rare.
Therefore, this study aims to investigate the effect of IFC treatment on pain, disability, and balance in patients with low back pain during functional movement.

\section{Materials and Methods}

\section{Participants}

40 patients with Low Back Pain (LBP) aged between 19 and 40 years were recruited for this study from M Hospital in Seoul. The subjects included in the study experienced low back pain for 3 months or longer, had a visual analogue scale score of 4 or higher, could maintain their standing posture independently for 30 seconds or longer, and were able to understand and follow the instructions given by the researcher (MMSE $>24$ points). The following patients were excluded: patients with radiculopathy; those with contraindications to electrotherapy, lumbar fracture or surgery; those taking medications that can affect the posture control, gait, or pain, lumbar-related diseases; those who received any physical therapy 8 weeks before the recruitment; and those taking corticosteroids 2 weeks before recruitment in this study [14]. Table 1 shows the common characteristics of the participants in this study. Informed consent was voluntarily obtained from all patients before participation in this study, which was approved by the Institutional Review Board (IRB) of Gachon University (IRB no. 1044396-201801-HR-009-01). This study used

Table 1. General Characteristics of the Participants

\begin{tabular}{cccc}
\hline Variable & IFC Group $(\mathrm{n}=20)$ & Placebo Group $(\mathrm{n}=20)$ & $\mathrm{p}$-value \\
\hline Sex & & & \\
Male & 7 & 8 & $.540^{\mathrm{a}}$ \\
Female & 13 & 12 & \\
Age & $56.42 \pm 10.45$ & $53.26 \pm 16.24$ & $.416^{\mathrm{b}}$ \\
Weight $(\mathrm{kg})$ & $63.24 \pm 17.62$ & $64.3 \pm 18.80$ & $.728^{\mathrm{b}}$ \\
Height $(\mathrm{cm})$ & $164.63 \pm 16.01$ & $167.1 \pm 14.73$ & $.605^{\mathrm{b}}$ \\
\hline
\end{tabular}

MMSE, Mini-mental Status Examination; VAS, Visual Analogue Scale; ODI, Oswestry Disability Index for Low Back Pain

${ }^{\text {a }}$; Chi-square Test ${ }^{\mathrm{b}}$; Indepentt-test

Values are Expressed as Mean \pm Standard Deviation (SD) 
the power analysis software G-power (version 3.1.7, Heinrich Heine University, Dusseldorf, Germany). for calculation of the sample size, which was determined on the basis of ability to detect a clinically significant improvement in the outcome measures from a pilot study (10 patients with IFC group and 10 patients with placebo group), and set the effect size as .8 and the alpha error as .05. According to the analysis, at least 20 subjects for each group were necessary to make an acceptable group size.

\section{Experimental Procedure}

This study was designed as a double-blinded randomized controlled trial (RCT). The participants were evaluated before and one day after training for two weeks by three well-trained physical therapists, who were not informed on the subjects and the purpose of this study. Forty patients selected by the inclusion criteria were randomized to minimize selection bias into IFC group $(\mathrm{n}=20)$ and placebo IFC $(n=20)$ group. A person who was not involved in the study picked out a number (either 1 or 2) from a sealed envelope for unbiased randomization. The treatment lasted 30 minutes a day, five times a week for two weeks. Subjects in the IFC treatment group received electrical stimulation for 30 minutes while those in the placebo treatment group received non-electrically stimulated IFC for same amount of time. All of the subjects received conventional therapy for an hour a day, five times a week for two weeks. Conventional therapy included hot pack therapy and trunk muscle stretching.

\section{Intervention}

Subjects were asked to expose their waist in a prone position, and they were electrically stimulated for 30 minutes using an IFC treatment device (IF - 7P; ITO CO., Japan). Four electrodes were attached so that they are crossed at the lumbar 1 and 5 height. The carrier frequency was set to $4000 \mathrm{~Hz}$ and the amplitude modulated frequency to $80 \mathrm{~Hz}$. The current electricity intensity was adjusted to an individual tolerable level of pins-and-needles sensation at a level of invisible muscle contraction. Furthermore, the mediator was asked to observe whether motion due to muscle contraction occurred in the participant. Electrodes were attached at the same location, but electrical stimulation was not applied in the placebo treatment group.

\section{Outcome Measures}

The Visual Analogue Scale (VAS) was used to measure pain. The score for each item ranges from 0 to 10 , measured in units of length up to the location where the patient indicated the level of pain. VAS is known to have a high sensitivity to pain and is proportional to the pain. This is the most widely used measurement method for chronic pain as well as acute pain with a good reliability [23]. In this study, resting pain and pain experienced during trunk forward flexion in the standing position and arm reaching out to a dominant side in the sitting position were measured. After waking up in the morning before and after training, the intensity of the pain was measured in a self-reported form while sitting still, and the intensity of the pain was measured when the waist was bent at the maximum possible angle in the standing position. In addition, pain intensity was measured when the dominant arm was extended diagonally in a sitting position. The maximum possible waist flexion angle and reaching distance were measured and applied equally before and after training.

The Oswestry Disability Index (ODI) was used for functional assessment of low back pain. ODI is a self-administered questionnaire consisting of 10 items, including pain intensity, sleep quality, ability to care for oneself, ability to walk, ability to sit, ability to stand, lifting, sexual function, social life and ability to travel and each item is described in 6 steps from score 0 to score 5 . The index provides a score out of 50, which in turn may be calculated as a percentage of disability value $(0 \%-100 \%)$ 
[24]. The test-retest reliability of ODI is .92 which is very high [25], and the categories are divided into $0-10 \%$ minimally disabled, $20-40 \%$ moderately disabled, $40-60 \%$ severely disabled, $60-80 \%$ crippled and $80-100 \%$ bedbound [26]. A force plate (PDM Multifunction Force Measuring Plate; Zebris, Germany, 2015) was used to measure the postural sway. After initialization of the force plate before the measurement, the postural sway was measured for 30 seconds with eyes open and with eyes closed in a comfortable posture with both arms placed on the outside of the leg on the force plate. The average value was obtained after each measurement was repeated 3 times.

\section{Data Analysis}

Normality of variables were assessed using the Shapiro-Wilk test. Independent t-test (for continuous variables) and the chi-square test (for categorical variables) were used for comparison of the baseline characteristics of subjects in the IFC and placebo stimulation groups. Paired t-test was used to compare changes to the baseline within the group and independent t-test was used for comparison between groups for the amount of change before and after. The level of statistical significance was set at .05 . SPSS 21.0 was used for statistical analysis.

\section{Results}

No one was dropped out after the training. There were no serious problems, such as cutaneous inflammation, pain and infection, following training.

No significant difference was found in general characteristics between the IFC and placebo stimulation groups before treatment (Table 1). After training, the VAS during the anterior trunk flexion in a standing position was significantly decreased in the IFC group compared to the placebo stimulation group. The changeable amount before and after training was $1.00 \pm 1.03$ and $.30 \pm .92$ point, respectively. No significant differences were observed pain at rest and pain experienced during reaching out in a sitting position between the IFC and placebo stimulation groups. ODI score significantly decreased in the IFC group (mean change, $2.85 \pm .89$ points) compared with the placebo stimulation group (mean change, $1.30 \pm 2.27$ points). The treatment caused a more significant decrease in postural sway when subjects stood with their eyes closed in the IFC group (mean change, $77.87 \pm 69.77$ ) than in the placebo stimulation group (mean change, $27.36 \pm 44.82 \mathrm{~cm}$ ). No significant differences were found in postural sway when subjects stood with their eyes opened between the IFC and placebo stimulation groups (Table 2).

\section{Discussion}

This study examined the immediate effects of IFC application on pain and disability in patients with chronic nonspecific low back pain. As a result, pain and disability were significantly reduced in the IFC group compared to the placebo stimulation group. Fuentes et al. [27] reported that a single application of IFC to chronic low back pain increased the threshold of press pain, and that the pain perception and disability level were significantly improved in the IFC-applied study for 2 weeks for low back pain patients [14]. The analgesic effect of IFC therapy can be explained by the Wedensky inhibition of type $\mathrm{C}$ nociceptive fibers and the action potential of large-diameter myelinated afferent nerve. The action potential of large-diameter myelinated afferent nerve starts from the cutaneous receptors and competes with a small diameter non-myelinated afferent nerve that transmits pain information to go to the central ascending sensory tract in the dorsal horn of the spinal cord. When $\mathrm{A} \beta$ fibers are excited by IFC, they cause pain reduction by blocking the pain information of $\mathrm{C}$ fibers entering dorsal horn [11]. However, when Correa et al. [13] examined the effects of IFC on resting pain and pain during sit to stand for inducing pain, there were no significant differences measured and it has been reported 
Table 2. Comparison of Experimental Group and Control Group

\begin{tabular}{cccccccc}
\hline \multirow{2}{*}{ Test } & \multirow{2}{*}{ Group } & \multicolumn{2}{c}{ VAS } & & \multicolumn{2}{c}{ Postural Sway Distance (cm) } \\
\cline { 3 - 8 } & & Resting & Trunk Anteflexion Reaching Out & ODI(\%) & Eyes Open & Eyes Closed \\
\hline \multirow{2}{*}{ Pre } & IFC Group & $6.50 \pm 1.32$ & $7.95 \pm .89$ & $7.75 \pm .72$ & $48.65 \pm 9.20$ & $285.45 \pm 180.60$ & $299.12 \pm 199.88$ \\
& Placebo Group & $6.80 \pm 1.06$ & $7.50 \pm 1.05$ & $7.35 \pm .88$ & $48.35 \pm 8.39$ & $261.00 \pm 121.07$ & $293.08 \pm 160.71$ \\
\multirow{2}{*}{ Post } & IFC Group & $6.10 \pm 1.25$ & $6.95 \pm 1.00$ & $6.90 \pm .97$ & $45.80 \pm 8.37$ & $245.95 \pm 117.49$ & $221.25 \pm 142.04$ \\
& Placebo Group & $6.45 \pm 1.39$ & $7.20 \pm 1.20$ & $6.80 \pm 1.01$ & $47.05 \pm 8.29$ & $244.17 \pm 108.88$ & $265.72 \pm 154.15$ \\
Amount & IFC group & $-.40 \pm 1.23$ & $-1.00 \pm 1.03^{*}$ & $-.85 \pm .75^{*}$ & $-2.85 \pm .89^{*}$ & $-39.50 \pm 91.95$ & $-77.87 \pm 69.77^{*}$ \\
of Change & Placebo Group & $-.35 \pm .93$ & $-.30 \pm .92^{*}$ & $-.55 \pm .69^{*}$ & $-1.30 \pm 2.27$ & $-16.83 \pm 78.27$ & $-27.36 \pm 44.82^{*}$ \\
p-value & & .886 & .029 & .193 & .039 & .406 & .010 \\
\hline
\end{tabular}

VAS, Visual Analogue Scale; ODI, Oswestry Disability Index for Low Back Pain, Values are Expressed as Mean \pm Standard Deviation (SD)

the obtained result is invalid because the sit-to-stand is not an appropriate movement for measuring pain in the case of low back pain as the pain level during sit-to-stand was lower than even the case of resting pain. Therefore, in this study, we compared the pain experienced during anterior trunk flexion in the standing position and arm reaching out in the sitting position, as well as the resting pain. During anterior trunk flexion in the standing position, IFC treatment significantly decreased pain compared to the placebo group. During functional movement, the pain increased more than that experienced in the resting state due to the trunk muscle contraction and change in the spinal alignment, and it is thought that there was a significant decrease in pain on IFC treatment during the anterior trunk flexion in the standing position, which is the posture involving maximal movement in the lumbar region. In a study using IFC in conjunction with lumbar region massage, it was reported that resting pain intensity, disability, and quality of life was improved [28]. In the present study, we also found that after IFC application, there was a significant improvement in the measurement of disability, and it is thought that this improvement is due to the improvement in pain during functional performance as well as in the resting pain.

This study also investigated the effect of IFC treatment on postural sway. As a result, there was no difference between the groups with the eyes open, but there was a significant reduction effect with the eyes closed. Patients with LBP increased postural agitation due to impaired intrinsic perception $[5,6]$ In this study, I think the decrease in posture fluctuation after IFC treatment was due to the improvement in the sense of intrinsic acceptance after training. Electrical stimulation has been reported to improve postural sway because it provides a cutaneous and proprioceptive sensory input that contributes to the recognition of vertical posture [29]. Also, Suh et al. [12] reported that the application of IFC to calf muscle for stroke patients resulted in a significant decrease in postural sway, and somatosensory input by IFC is thought to have increased the excitability of motor cortex. In the case of TENS applied to the human body by generating currents similar to IFC, the instability of the sitting posture was improved by applying TENS to the neck muscles of the stroke patients [29], and $\mathrm{Ng} \&$ Hui-Chan et al. [30] reported that TENS in patients with hemiplegia improved balance-related gait function. Although it is not the same electrical stimulation method as TENS, it is thought that electric stimulation caused by IFC improves posture control by increasing sensory input as in the case of TENS. This study examined the effects of IFC on pain, disability, and 
balance in patients with chronic nonspecific low back pain. However, the number of subjects was small, and the treatment period was short. Also, it was not confirmed whether intrinsic acceptance was improved through training. therefore, for future studies it is necessary to evaluate the effects of the long-term treatment. In addition, the behavioral analysis will be useful for investigating the change in a sitting posture, as well as pain and balance when IFC is combined with exercise.

\section{Acknowledgements}

This work was supported by the 2019 Gimcheon University Research Grant.

This work was supported by the National Research Foundation of Korea (NRF) grant funded by the Korea government (MSIT) (No. 2017R1C1B507659714).

\section{References}

[1] Vos T, Flaxman AD, Naghavi M, et al. Years lived with disability (YLDs) for 1160 sequelae of 289 diseases and injuries 1990-2010: a systematic analysis for the Global Burden of Disease Study 2010. Lancet. 2012; 380(9589):2163-96.

[2] Ekman M, Johnell O, Lidgren L. The economic cost of low back pain in Sweden in 2001. Acta Orthop. 2005;76(2):275-84.

[3] Liu-Ambrose T, Eng JJ, Khan KM, et al. The influence of back pain on balance and functional mobility in 65-75-year-old women with osteoporosis. Osteoporos Int. 2002; 13(11):868-73.

[4] Baratto L, Morasso PG, Re C, et al. A new look at posturographic analysis in the clinical context: swaydensity versus other parameterization techniques. Mot Control. 2002;6(3):246-70.

[5] Brumagne S, Cordo P, Lysens R, et al. The role of paraspinal muscle spindles in lumbosacral position sense in individuals with and without low back pain. Spine (Phila Pa 1976). 2000;25(8):989-94.

[6] Casadio M, Morasso PG, Sanguineti V. Direct measurement of ankle stiffness during quiet standing: implications for control modelling and clinical application. Gait Posture. 2005;21(4):410-24.

[7] Facci LM, Nowotny JP, Tormem F, et al. Effects of transcutaneous electrical nerve stimulation (TENS) and interferential currents (IFC) in patients with nonspecific chronic low back pain: Randomized clinical trial. Sao Paulo Med J. 2011;129(4):206-16.

[8] Pantaleão MA, Laurino MF, Gallego NL, et al. Adjusting pulse amplitude during transcutaneous electrical nerve stimulation (TENS) application produces greater hypoalgesia. J Pain. 2011;12(5):581-90.

[9] Bae YH, Lee SM. Analgesic effects of transcutaneous electrical nerve stimulation and interferential current on experimental ischemic pain models: Frequencies of 50 Hz and 100 Hz. J Phys Ther Sci. 2014;26(12):1945-8.

[10] Hurley DA, Minder PM, McDonough SM, et al. Interferential therapy electrode placement technique in acute low back pain: a preliminary investigation. Arch Phys Med Rehabil. 2001;82(4):485-93.

[11] Hurley DA, McDonough SM, Dempster M, et al. A randomized clinical trial of manipulative therapy and interferential therapy for acute low back pain. Spine 2004;29(20):2207-16.

[12] Suh HR, Han HC, Cho HY. Immediate therapeutic effect of interferential current therapy on spasticity, balance, and gait function in chronic stroke patients: a randomized control trial. Clin Rehabil. 2014;28(9):885-91.

[13] Corrêa JB, Costa LO, Oliveira NT, et al. Effects of the carrier frequency of interferential current on pain modulation and central hypersensitivity in people with chronic nonspecific low back pain: A randomized placebo-controlled trial. Eur J Pain. 2016;20(10):1653-66.

[14] Albornoz-Cabello M, Maya-Martín J, DomínguezMaldonado G, et al. Effect of interferential current therapy 
on pain perception and disability level in subjects with chronic low back pain: a randomized controlled trial. Clin Rehabil. 2017;31(2):242-9.

[15] Werners R, Pynsent PB, Bulstrode CJ. Randomised trial comparing interferential electrotherapy with motorized lumbar traction and massage in the management of low back pain in a primary care setting. Spine. 1999;24(15): 1579-84.

[16] Olaogun RA, Oyeyemi MO, Oyeyemi AL. Transcutaneous electrical nerve stimulation and interferential current combined with exercise for the treatment of knee osteoarthritis: a randomised controlled trial. Hong Kong Physiother J. 2005;23(1):13-9.

[17] Zambito A, Bianchini D, Gatti D, et al. Interferential and horizontal therapies in chronic low back pain: a randomized, double blind, clinical study. Clin Exp Rheumatol. 2006;24(5):534-9.

[18] Van der Heijden GJ, Leffers P, Wolters PJ, et al. No effect of bipolar interferential electrotherapy and pulsed ultrasound for soft tissue shoulder disorders: a randomized controlled trial. Ann Rheum Dis. 1999;58(9):530-40.

[19] Cheing GL, So EM, Chao CY. Effectiveness of electroacupunture and interferential electrotherapy in the management of frozen shoulder. J Rehabil Med. 2008; 40(3):166-70.

[20] Taskaynatan MA, Ozgul A, Ozdemir A, et al. Effects of steroid iontophoresis and electrotherapy on bicipital tendonitis. J Musculoskelet Pain. 2007;15(4):47-54.

[21] Serrano-Atero MS, Caballero J, Canas A, et al. Pain assessment (I). Rev Soc Esp Dolor. 2002;9: 94-108.

[22] Thiese MS, Hughes M, Biggs J. Electrical stimulation for chronic non-specific low back pain in a working-age population: a 12-week double blinded randomized controlled trial. BMC Musculoskelet Disord. 2013;14: 117.

[23] Yarnitsky D, Sprecher E, Zaslansky R. Multiple session experimental pain measurements. Pain. 1996;67(2-3): 327-33.

[24] Alcantara-Bumbiedro S, Florez-Garcia MT, EchavarriPerez C, et al. Escala de incapacidad por dolor lumbar de Oswestry. Rehabilitación (Madr) 2006;40(3): 150-8.

[25] Kim DY, Lee SH, Lee HY, et al. Validation of the Korean version of the Oswestry disability index. Spine (Phila Pa 1976). 2005;30(5):E123-7.

[26] Miralles RC, Rull M. Valoración de los resultados del tratamiento del dolor lumbar y de las secuelas. Rev Soc Esp Dolor. 2001;8(Supl. II):131-9

[27] Fuentes J, Armijo-Olivo S, Funabashi M et al. Enhanced therapeutic alliance modulates pain intensity and muscle pain sensitivity in patients with chronic low back pain: an experimental controlled study. Phys Ther. 2014;94(4): 477-89.

[28] Lara-Palomo IC, Aguilar-Ferrandiz ME, MataranPenarrocha GA, et al. Short-term effects of interferential current electro-massage in adults with chronic nonspecific low back pain: a randomized controlled trial. Clin Rehabil. 2013;27(5):439-49.

[29] Perennou DA, Leblond C, Amblard B, et al. Transcutaneous electric nerve stimulation reduces neglect-related postural instability after stroke. Arch Phys Med Rehabil. 2001;82(4):440-48.

[30] Ng SS, Hui-Chan CW. Does the use of TENS increase the effectiveness of exercise for improving walking after stroke? A randomized controlled clinical trial. Clin Rehabil. 2009;23(12):1093-103. 